\title{
Record Mood
}

National Cancer Institute

\section{Source}

National Cancer Institute. Record Mood. NCI Thesaurus. Code C117051.

The modes that may be applied to a record as it passes through various phases of a life cycle or business process. 